\title{
Starry messages
}

\author{
The first scientific observations with telescopes displaced Earth from the centre of the Universe. \\ Modern technology continues to humble us but should not distance us from the cosmos itself.
}

$\Gamma_{\text {ti }}$ ou our hundred years ago, the Universe changed. Or, at least, our perception of it did, thanks to Galileo Galilei's scrutiny of the night sky with a telescope. Within a couple of years, his observations of the Moon, phases of Venus and satellites of Jupiter shattered the old Ptolemaic model of our Solar System. To the church's dismay, Earth assumed its rightful place as one of several planets orbiting the Sun (see page 28).

Marking Galileo's anniversary, the International Year of Astronomy seeks to remind us of the humbling nature of gazing at the heavens. Through programmes offering cheap telescopes for the masses, teaching materials and global heritage projects, astronomers are inviting us all to look up and ponder our place in the Universe.

That this prospect is novel speaks to the distance that has come between people and their environment. Urban light pollution means that one-fifth of the world's population can no longer see the Milky Way with the naked eye. Many city kids, even if they did peer through the orange smog above their heads, would probably see only a handful of stars. Astronomers are calling for the dark places on Earth to be preserved as national parks, so that we do not lose completely our window on the Universe (see page 27).

Astronomers, in communicating their work, have a natural ally in beautiful images. The Hubble Space Telescope has a place in the public's heart for this reason - although it has accomplished outstanding science too (see page 41). But professional astronomers themselves are not immune to distancing. The technological advances that have given so much insight into astrophysics have made astronomy one of the most computer-intensive of the natural sciences. Surveys churn out terabytes of data to be crunched by armies of postdocs and students

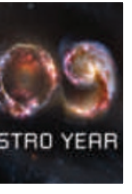

who might never have visited a mountain-top observatory. Could data mining soon replace serendipitous observational discovery? Astronomers will anyway need new visualization tools, as demonstrated by a three-dimensional graphic in this issue (see page 63), to hunt for the unexpected.

Since Galileo, advancing telescope technology has made our human perspective seem ever more myopic. We now know that the Milky Way is but one of many galaxies scattered throughout the Universe. Space is littered with fearsome objects, from giant black holes to energetic $\gamma$-ray bursts. Planets are plentiful around other stars. Even "One-fifth of the world's population can no longer see the Milky Way with the naked eye." the normal matter that makes up our bodies is only a minority constituent of the Universe - most matter is exotic and dark.

The next generation of telescopes (see page 18) will push astronomy into a new realm. Within two decades the whole night sky will be scanned continuously and recorded digitally across many wavebands. Trips to observatories will become obsolete, except for detailed followup work. Astronomical data will be generated on an industrial scale and mined from a laptop. Yet the new vistas revealed will still surprise.

Every now and then, someone predicts the 'end of science.' It was believed in the 1890s that there were just a few problems left to solve in physics: yet Albert Einstein solved three of them in 1905, and founded three new branches of physics. In the next 40 years, never mind the next 400, astronomy will change. It is not the end, but the beginning of a new phase. The Universe will continue to humble us, if we take the time to look.

\section{Experts still needed}

\section{There are good reasons to be suspicious of metric-based research assessment.}

M any countries are keen to measure their universities' research performance with minimal burdens on the participants. Not least of these is the United Kingdom, which last month announced the results of its sixth and final Research Assessment Exercise (RAE, see page 13).

The RAE relied heavily on expert peer review of research publications, and attention in Britain and beyond is now focused on what form the replacement system will take. The proposed successor, the Research Excellence Framework (REF), is opaque. Little is known about how it will work other than a central principle: it will assess research quality using metrics, including publication citations. It may also take into account the number of postgraduates completing their studies and the amount of research income won by universities. There will be a smattering of 'light-touch expert review', although the exact form that this will take is not yet clear - it might simply be used to interpret the metrics results.

But taken alone, publication citations have repeatedly been shown to be a poor measure of research quality. An example from this journal illustrates the point. Our third most highly cited paper in 2007, with 272 citations at the time of inspection, was of a pilot study in screening for functional elements of the human genome. The importance lay primarily in the technique. In contrast, a paper from the same "Metrics are not well established for the applications of science." year revealing key biological insights into the workings of a proton pump, which moves protons across cell membranes, had received 10 citations. There are plenty more examples of such large disparities between papers that may be important for a variety of reasons: technological breakthroughs of immediate use to many, more rarefied achievements of textbook status, critical insights of relevance to small 